\title{
Morphology of the Insula in Relation to Hearing Status and Sign Language Experience
}

\author{
John S. Allen, ${ }^{1,2}$ Karen Emmorey, ${ }^{3}$ Joel Bruss, ${ }^{4}$ and Hanna Damasio ${ }^{1,2}$ \\ ${ }^{1}$ Dornsife Cognitive Neuroscience Imaging Center, University of Southern California, Los Angeles, California 90089-2520, ${ }^{2}$ Brain and Creativity Institute, \\ University of Southern California, Los Angeles, California 90089-1061, ${ }^{3}$ Laboratory for Language and Cognitive Neuroscience, San Diego State University, \\ San Diego, California 92120, and ${ }^{4}$ Department of Neurology, Laboratory of Computational Neuroimaging, University of Iowa Carver College of Medicine, \\ Iowa City, Iowa 52242
}

We investigated whether auditory deprivation and/or sign language exposure during development alters the macroscopic neuroanatomy of the human insula. Volumetric analyses were based on MRI data from 25 congenitally deaf subjects who were native users of American Sign Language (ASL), 25 hearing subjects with no knowledge of ASL, and 16 hearing subjects who grew up in deaf families and were native ASL signers. Significant variation in insula volume was associated with both hearing status and sign language experience. Compared with both hearing groups, deaf subjects exhibited a significant increase in the amount of gray matter in the left posterior insular lobule, which we hypothesize may be related to the dependence on lip-reading and articulatory-based (rather than auditory-based) representations of speech for deaf individuals. Both deaf and hearing signers exhibited an increased volume of white matter in the right insula compared with hearing nonsigners. We hypothesize that the distinct morphology of the right insula for ASL signers may arise from enhanced connectivity resulting from an increased reliance on cross-modal sensory integration in sign language compared with spoken language.

Key words: insula; American Sign Language; deaf; gray matter; white matter; MRI

\section{Introduction}

The insula has an important integrative role in cortical circuitry and extensive afferent and efferent connections to a wide range of cortical and limbic areas (Augustine, 1996). It is involved in cognitive, emotional, autonomic, and sensory processes (Shelley and Trimble, 2004). The insula is parcellated into two portions by the central insular sulcus; the anterior portion consists of three "short" gyri, whereas the posterior comprises two "long" gyri (Türe et al., 1999). Cytoarchitectonically, the insula is divided into three sectors. The rostroventral insula consists of agranular cortex, the posterior insula is made up of granular cortex, and between these two sectors, there is a transitional dysgranular sector continuous with the agranular cortex (Mesulam and Mufson, 1982a; Flynn et al., 1999). Each sector has extensive connections with the frontal, temporal, and parietal lobes and the cingulate gyrus (Mesulam and Mufson, 1982b; Mufson and Mesulam, 1982). The insula is implicated in auditory processing and motor aspects of speech among other functions (Bamiou et al., 2003).

Neurocognitive studies of deaf and hearing individuals who are native users of American Sign Language (ASL) provide us with a unique perspective on how the environment influences the structurofunctional anatomy of the brain (Emmorey et al., 2003).

Received July 7, 2008; revised Sept. 20, 2008; accepted Sept. 30, 2008.

This work was supported by a grant from the National Institute on Deafness and Other Communication Disorders (R01 DC006708) awarded to K.E.

Correspondence should be addressed to Karen Emmorey, Laboratory for Language and Cognitive Neuroscience, San Diego State University, 6495 Alvarado Road Suite 200, San Diego, CA 92120. E-mail: kemmorey@mail.sdsu.edu. DOI:10.1523/JNEUROSCI.3141-08.2008

Copyright $\odot 2008$ Society for Neuroscience ～0270-6474/08/2811900-06\$15.00/0
Such investigations are taking on added importance as the plasticity of cortical networks is becoming increasingly evident (e.g., Majewska and Sur, 2006). In this study, we use high resolution magnetic resonance imaging to measure gray and white matter volumes of the insula in hearing and congenitally deaf ASL signers and in hearing nonsigners. There is both functional and structural evidence that supports the hypothesis that hearing status and/or experience with a spoken or signed language can affect the anatomy of the insula. The insula has extensive connections with primary and secondary auditory regions of the temporal lobe and to the speech areas of the frontal lobe (Shelley and Trimble, 2004). Lesion studies show that the anterior insula is important for speech articulation and motor planning (Dronkers, 1996; Ackermann and Riecker, 2004). Functional imaging indicates that the posterior insula is important in the auditory processing of speech; the insula may integrate different neural networks involved in speech production and perception (Noesselt et al., 2003). Sign language in deaf individuals activates both classical language areas and other regions including the posterior insula (Kassubek et al., 2004). Given auditory deprivation and differential experience with spoken language for deaf persons, we predict that differences in insular anatomy may arise between deaf and hearing individuals.

Many cortical regions play a role in multisensory integration (Driver and Noesselt, 2008), and functional imaging studies suggest that the insula/claustrum region is particularly important in cross-modal integration of conceptually related but sensorily independent stimuli (audiovisual or tactile-visual) (Hadjikhani and Roland, 1998; Banati et al., 2000; Bischoff et al., 2007; Naghavi et al., 2007). Sign language is a skill that involves tactile- 
visual integration, particularly when monitoring language output (Emmorey et al., 2008a,b). Speakers auditorily monitor their language output, but signers do not look directly at their hands and must integrate proprioceptive and tactile sensory information with visual input from the periphery of vision to monitor their language output. We therefore predict that insular morphology may vary according to sign language experience, regardless of hearing status.

\section{Materials and Methods}

Subjects. Subjects were 25 congenitally deaf individuals who were native ASL signers ( 14 women and 11 men, average age 23.8 years, SD 4.1, range 19-38), 25 hearing individuals with no knowledge of ASL (14 women and 11 men, average age 28.5 years, SD 4.5, range 22-39), and 16 hearing individuals who acquired ASL from their deaf parents ( 10 women and 6 men, average age 24.3 years, SD 4.4, range 19-38). Although the hearing nonsigners are slightly older than the other two groups, age should not be a significant contributor to variation in insular anatomy among these groups (Allen et al., 2005a,b). These hearing signers acquired expertise in ASL as children, concurrently with their acquisition of spoken language. The hearing nonsigners were monolingual English speakers with no history of hearing disorder. All subjects were right-handed, with scores on the Oldfield-Geschwind Handedness Inventory above +90 (maximum right-handed score +100 ). All subjects were healthy with no history of neurological or psychiatric illness. Twenty-one deaf subjects exhibited profound hearing loss ( $>90 \mathrm{~dB}$ in the better ear), three subjects had severe hearing loss ( $>75 \mathrm{~dB}$ in the better ear), and one subject had moderately severe hearing loss ( $>55 \mathrm{~dB}$ in the better ear). Data on early hearing aid use was available for 17 deaf subjects. None used a hearing aid consistently before the age of 2 . Eleven were required to wear hearing aids at school, but only two also wore hearing aids at home, and six subjects did not wear hearing aids at home or at school. All deaf subjects were congenitally deaf and were born to deaf parents, and ASL was their primary and first language. All subjects gave informed consent in accordance with institutional and federal rules.

MR image acquisition. Thin cut MR images were obtained in a GE Signa scanner operating at $1.5 \mathrm{~T}$ by using the following protocol: SPGR/ 50, TR 24, TE 7, NEX 1 matrix $256 \times 192$, FOV $24 \mathrm{~cm}$. We obtained 124 contiguous coronal slices, 1.5 or $1.6 \mathrm{~mm}$ thick and interpixel distance $0.94 \mathrm{~mm}$. Three individual 1NEX SPGR datasets were obtained for each subject during each imaging session. These were coregistered and averaged post hoc using Automated Image Registration (AIR 3.03, UCLA) (Woods et al., 1992; Holmes et al., 1998).

MR image analysis [3D reconstructions and volume determinations from regions of interest (ROIs)] were conducted using Brainvox (Frank et al., 1997), an interactive family of programs designed to reconstruct, segment, and measure brains from MR acquired images. An automated program, extensively validated against human experts (Grabowski et al., 2000), was used to segment the images into the three primary tissue types (white, gray, cerebral spinal fluid). Before tracing ROIs, brains were realigned (but not resized) along a plane running through the anterior and posterior commissures (i.e., the AC-PC line); this ensured that coronal slices in all subjects were perpendicular to a uniformly and anatomically defined axis of the brain.

Regions of interest. ROIs were traced by hand on contiguous coronal slices of the brain. Insula and cerebral hemisphere ROIs were traced as described previously (Allen et al., 2003; see Allen et al., 2005b, for insula reliability statistics). In brief, the insula is defined laterally by the circular sulcus and mesially by an arbitrary line linking the deepest extents of the two ends of the circular sulcus. If the claustrum interrupts this arbitrary line, it is edited out of the insula ROI. Thus the gray matter volume of the insula includes only insular cortex. The white matter volume includes all of the white matter between the claustrum and the insular cortex, corresponding approximately to the extreme capsule. The parcellation of the insula into the anterior and posterior insular lobules was done in a manner similar to that described by Makris et al. (2006). The central sulcus of the insula (CeSI) was used to divide the insula into its anterior and posterior parts. The CeSI was identified and marked on contiguous coro-
$\mathbf{A}$

GRAY MATTER PROPORTION

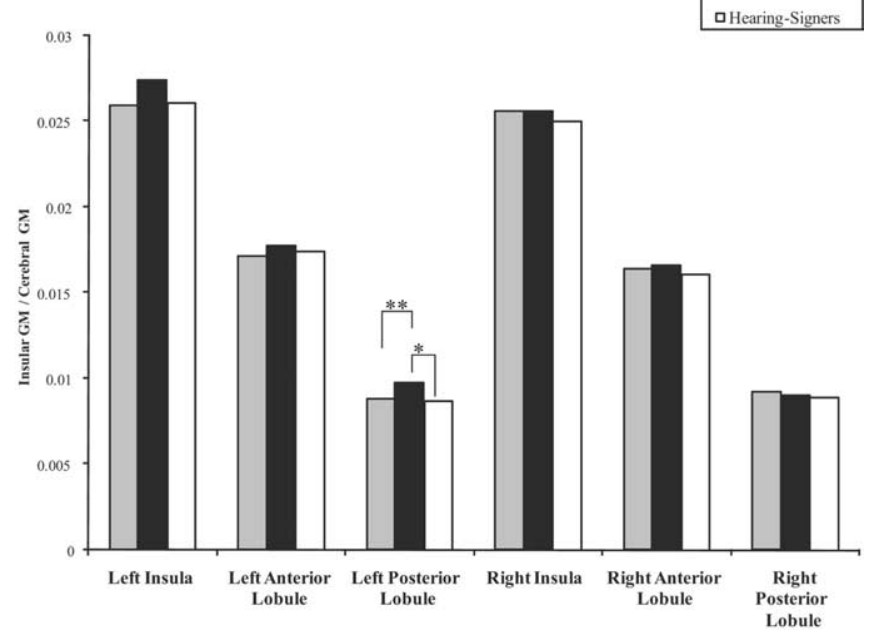

B

WHITE MATTER PROPORTION

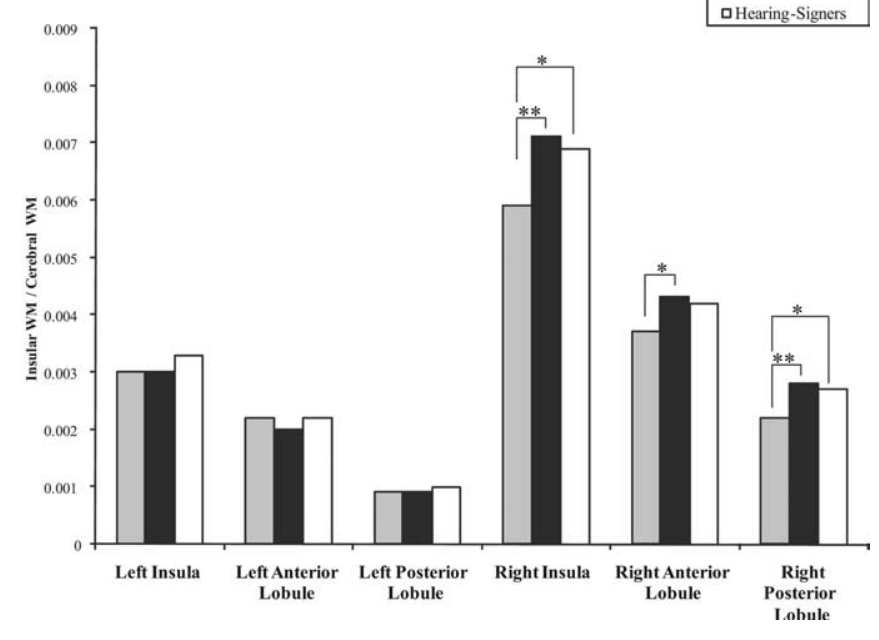

Figure 1. A, Insula gray and white matter proportional volumes by groups and region ( $t$ test, $\left.{ }^{*} p<0.05 ;{ }^{* *} p<0.01\right) . \boldsymbol{B}$, Insula white matter proportional volumes by groups and region ( $t$ test, ${ }^{*} p<0.05$; ${ }^{* *} p<0.01$ ).

nal and parasagittal slices of the brain through the insular region; the marked CeSI was then used to divide traced whole insula ROIs into anterior and posterior lobules. The division of the insula was confirmed by examining 3-D reconstructions of the anterior and posterior lobule ROIs (Fig. 1).

Data analysis. Statistical analyses were performed using SPSS 9.0.0 for Windows. Univariate ANOVA and Bonferroni-corrected $t$ tests were used to compare group means. Hemispheric asymmetries were examined with pairwise $t$ tests and a conventional asymmetry index $[(L-R) /[(L+R) / 2]]$.

\section{Results}

\section{Volumes and proportions}

The gray and white matter volumes of total insula and the anterior and posterior insular lobules are presented in Table 1. No group comparisons for these raw volumes are presented because the subject groups are not sex-matched, and there may be overall differences in brain size that could influence insula volume differences. Group differences are therefore examined using sizecorrected or size-neutral measures.

In Table 2, volume proportions of the insula are presented; the proportions consist of gray or white matter insula volume di- 
Table 1. Gray and white matter mean volumes (in $\mathrm{mm}^{3}$ ) of the insula by group (SD in parentheses)

\begin{tabular}{lcccr}
\hline Region & Tissue & Hearing & \multicolumn{1}{l}{ Deaf } & Hearing signers \\
\hline Left insula & GM & $7762(1095)$ & $8426(1182)$ & $7609(734)$ \\
& WM & $697(254)$ & $690(208)$ & $762(286)$ \\
Right insula & GM & $7762(1046)$ & $7998(1021)$ & $7366(755)$ \\
& WM & $1376(330)$ & $1644(324)$ & $1595(274)$ \\
Left anterior insula & GM & $5116(820)$ & $5426(808)$ & $5057(549)$ \\
& WM & $505(189)$ & $471(153)$ & $527(215)$ \\
Left posterior insula & GM & $2645(452)$ & $2999(522)$ & $2552(388)$ \\
& WM & $192(126)$ & $219(84)$ & $235(101)$ \\
Right anterior insula & GM & $4958(721)$ & $5181(702)$ & $4750(620)$ \\
& WM & $868(276)$ & $1003(231)$ & $979(209)$ \\
Right posterior insula & GM & $2804(494)$ & $2808(493)$ & $2616(262)$ \\
& WM & $508(117)$ & $640(172)$ & $616(109)$ \\
\hline
\end{tabular}

GM, Gray matter; WM, white matter.

vided by the hemisphere gray or white matter volume. Two patterns emerge here. First, for the gray volume proportion parcellation, there is only one significant difference: the deaf signers have significantly more gray matter in the left posterior lobule than either of the hearing groups (Fig. 1A). Second, for the white matter proportions, both the deaf and hearing signers have significantly more white matter in the right insula as a whole than the hearing nonsigners (Fig. $1 \mathrm{~B}$ ). This difference is significant for the deaf signers in both the anterior and posterior lobules; for the hearing signers the difference is significant for the posterior lobule, with a similar trend for the anterior lobule $(p=0.096)$.

\section{Gray matter/white matter ratios}

In Table 3, gray matter/white matter (GM/WM) ratios are presented for the whole insula and the anterior and posterior lobules. Two results stand out. First, for all subject groups and regions, the $\mathrm{G} / \mathrm{W}$ ratio is much higher for the left insula than for the right (Fig. 2 , Table 1); this is generally a result of having less white matter on the left side compared with the right, although there are group differences present within this overall trend (see following section). For the left posterior lobule, there is a significant difference between the hearing nonsigners and the hearing signers. Second, the increase in white matter in the right insula of the deaf and hearing signers reported in Table 2 and Figure $1 B$ is manifest in the consistently lower G/W ratios in the right insula for these subjects compared with the hearing nonsigners.

\section{Asymmetry index}

The asymmetry results are presented in Table 4. For the whole insula GM, there are significant differences between the hearing subjects and the deaf and hearing signers (Fig. 3A), with the hearing subjects being essentially symmetrical and the deaf and hearing signing subjects being moderately leftwardly asymmetric. However, the parcellation indicates that the anterior and posterior lobules have some different asymmetry patterns. For the anterior lobules, all subject groups have a rightward asymmetry, although this is more pronounced in the deaf and hearing signers. For the posterior lobule, the deaf signers are differentiated from the other two groups by having a leftward as opposed to rightward asymmetry. This again relates to the fact that the deaf signers have an increase in GM volume relative to the other two groups (Table 2).

For the WM, all subject groups have a pronounced rightward asymmetry. Compared with the hearing subjects, the rightward asymmetry is more pronounced in the deaf, especially the anterior lobule. The hearing signers appear to be intermediate be- tween the deaf and hearing subjects, especially for the anterior lobule.

Pairwise $t$ tests, which compare the left and right volumes within subjects, generally indicate that the AI scores reflect significant biases on a case-by-case basis (results not shown).

\section{Discussion}

The insula plays a key role in many complex cognitive processes. Given the great range of functions that involve the insula, one might predict that differences in any single cognitive domain would be unlikely to result in volumetric variation of the insula or one of its component lobules. Congenital deafness, however, represents a profound departure from the normative environment of brain development, and it may have multiple effects on different sensory and cognitive modalities (Bavelier et al., 2006). Furthermore, in hearing signers, the acquisition of ASL as a first language in conjunction with spoken language may also influence brain structure. We found that significant variation in insula volume is associated with both hearing status and sign language experience: compared with either of the hearing groups, deaf subjects have a significant increase in the amount of GM in the left posterior insular lobule; both deaf and hearing signers have an increased volume of WM in the right insula compared with hearing nonsigners.

Before looking at the differences associated with deafness or sign language experience, two more general issues need to be addressed. First, our parcellation of the insular lobules indicates that the volume of the anterior lobule is approximately twice that of the posterior (Tables 1,2). This ratio is similar to those reported in recent parcellations of the insula (Makris et al., 2006; Saze et al., 2007). The second issue involves the strong rightward asymmetry we observed for all subject groups in insula WM volume (Table 4, Fig. 3). We have observed this pattern in a larger normative sample of right-handed individuals, and discussed it at some length (Allen et al., 2003). It is important to note that comparing right and left insula WM volumes may be somewhat misleading because the mesial boundary of the insula ROI is defined as a line linking the anterior and posterior ends of the circular sulcus (as observed in a coronal plane), excluding the claustrum. Differences in the shape of the insular cortex could influence the amount of WM (i.e., the proportion of the extreme capsule located between the insula and claustrum) included in our insula ROI. That said, we suggest that the WM asymmetry results do indicate an asymmetry in the anatomy of the insula.

In a diffusion tensor imaging study, Cao et al. (2003) reported that the relative anisotropy of water diffusion is markedly higher in the left subinsular WM compared with the right; they suggest that this may reflect a difference in the orientation and organization of white matter fiber tracts in the subinsular region. Whatever its exact cause or function [Cao et al. (2003) relate it to language], it likely reflects a marked asymmetry in the insular white matter. Cao et al. (2003) did not find an asymmetry in subinsular WM volume, which they defined as all the WM between the insular cortex and the putamen; this was a more expansive definition of the insula WM than the one we used. There may be differences in the distribution of WM in the external and extreme capsules surrounding the claustrum, which could explain why we found an asymmetry, whereas Cao et al. (2003) did not. Even in the context of this asymmetry, we found increased WM volume in the right insula of the deaf and hearing signers compared with the hearing nonsigners. Thus, although left-right differences in WM volume should be regarded with caution, we 
Table 2. Gray and white matter insula volume proportions for anterior and posterior insular lobules by group

\begin{tabular}{|c|c|c|c|c|c|}
\hline \multirow[b]{2}{*}{ Proportion } & \multicolumn{3}{|c|}{ Volume proportion group mean (SD) } & \multirow[b]{2}{*}{ Between-groups $p$ value $\left(F_{(2,63)}\right)$} & \multirow[b]{2}{*}{ Significant differences (Bonferroni $p$ value) } \\
\hline & Hearing $(\mathrm{H})$ & Deaf & Hearing signers (HS) & & \\
\hline Left insula/hemi gray matter & $0.0259(0.0028)$ & $0.0274(0.0021)$ & $0.0261(0.0014)$ & $0.044(3.275)$ & Deaf $>\mathrm{H}(0.056)$ \\
\hline Left anterior lobule/hemi gray matter & $0.0171(0.0023)$ & $0.01767(0.0018)$ & $0.0174(0.0016)$ & ns & na \\
\hline Left posterior lobule/hemi gray matter & $0.0088(0.0012)$ & $0.0097(0.0011)$ & $0.0087(0.0008)$ & $0.003(6.362)$ & $\begin{array}{l}\text { Deaf }>H(0.008) \\
\text { Deaf }>H S(0.014)\end{array}$ \\
\hline Right insula/hemi gray matter & $0.0256(0.0027)$ & $0.0256(0.0020)$ & $0.0250(0.0015)$ & ns & na \\
\hline Right anterior lobule/hemi gray matter & $0.0164(0.0022)$ & $0.0166(0.0015)$ & $0.0161(0.0014)$ & ns & na \\
\hline Right posterior lobule/hemi gray matter & $0.0092(0.0011)$ & $0.0090(0.0013)$ & $0.0089(0.0007)$ & ns & na \\
\hline Left insula/hemi white matter & $0.0030(0.0011)$ & $0.0030(0.0006)$ & $0.0033(0.0011)$ & ns & na \\
\hline Left anterior lobule/hemi white matter & $0.0022(0.0007)$ & $0.0020(0.0005)$ & $0.0022(0.0008)$ & ns & na \\
\hline Left posterior lobule/hemi white matter & $0.0009(0.0009)$ & $0.0009(0.0003)$ & $0.0010(0.0004)$ & ns & na \\
\hline Right insula/hemi white matter & $0.0059(0.0011)$ & $0.0071(0.0011)$ & $0.0069(0.0010)$ & $<0.001(8.667)$ & $\begin{array}{l}\text { Deaf }>\mathrm{H}(<0.001) \\
\mathrm{HS}>\mathrm{H}(0.023)\end{array}$ \\
\hline Right anterior lobule/hemi white matter & $0.0037(0.0009)$ & $0.0043(0.0007)$ & $0.0042(0.0008)$ & $0.031(3.662)$ & Deaf $>\mathrm{H}(0.034)$ \\
\hline Right posterior lobule/hemi white matter & $0.0022(0.0005)$ & $0.0028(0.0007)$ & $0.0027(0.0004)$ & $0.002(7.124)$ & $\begin{array}{l}\text { Deaf }>\mathrm{H}(0.002) \\
\mathrm{HS}>\mathrm{H}(0.048)\end{array}$ \\
\hline
\end{tabular}

Hemi, Hemisphere.

Table 3. Gray matter/white matter ratios for the insula and insular lobules

\begin{tabular}{|c|c|c|c|c|c|}
\hline \multirow[b]{2}{*}{ Region } & \multicolumn{3}{|c|}{ GM/WM ratio group mean (SD) } & \multirow[b]{2}{*}{ Between-groups $p$ value $\left(F_{(2,63)}\right)$} & \multirow[b]{2}{*}{ Significant differences (Bonferroni $p$ value } \\
\hline & Hearing $(\mathrm{H})$ & Deaf & Hearing signers (HS) & & \\
\hline Left insula GM/WM & $12.35(3.85)$ & $12.91(2.76)$ & $11.17(3.86)$ & ns & na \\
\hline Left anterior lobule GM/WM & $11.41(4.09)$ & $12.38(3.29)$ & $11.12(4.63)$ & ns & na \\
\hline Left posterior lobule GM/WM & $16.65(5.84)$ & $14.98(4.32)$ & $12.58(4.85)$ & $0.050(3.152)$ & $\mathrm{H}>\mathrm{HS}(0.044)$ \\
\hline Right insula GM/WM & $5.87(1.20)$ & $4.97(0.77)$ & $4.75(0.98)$ & $0.001(7.657)$ & $\begin{array}{l}H>\operatorname{deaf}(0.007) \\
H>H S(0.003)\end{array}$ \\
\hline Right anterior lobule GM/WM & $6.14(1.61)$ & $5.32(0.88)$ & $5.06(1.24)$ & $0.020(4.183)$ & $\mathrm{H}>\mathrm{HS}(0.032)$ \\
\hline Right posterior lobule GM/WM & $5.66(1.06)$ & $4.55(0.96)$ & $4.36(0.83)$ & $<0.001(11.814)$ & $\begin{array}{l}\mathrm{H}>\operatorname{deaf}(<0.001) \\
\mathrm{H}>\mathrm{HS}(<0.001)\end{array}$ \\
\hline
\end{tabular}

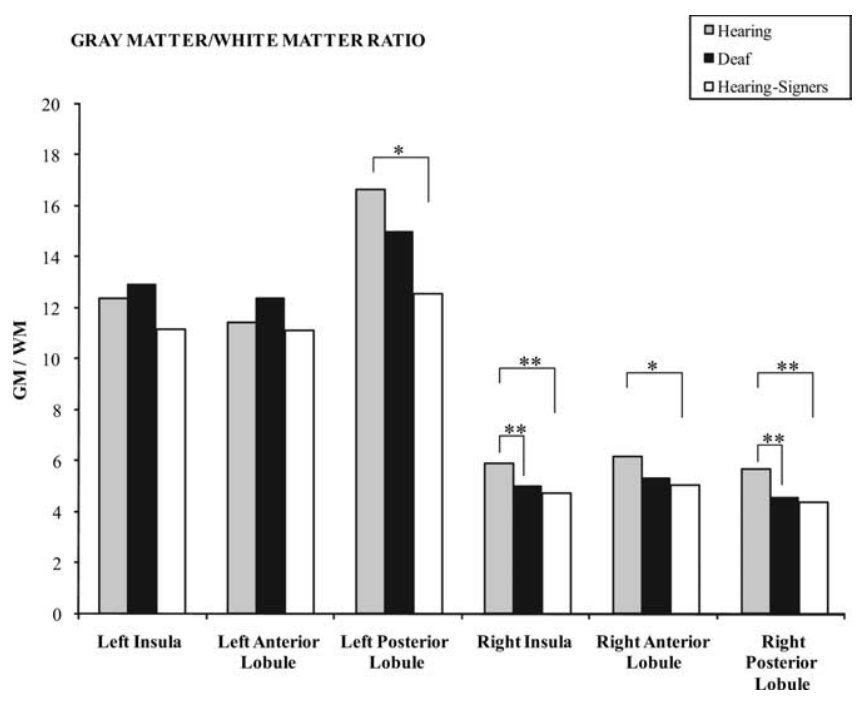

Figure 2. Insula gray matter/white matter ratios by region $\left(t\right.$ test, $\left.{ }^{*} p<0.05 ;{ }^{* *} p<0.01\right)$.

suggest that unilateral between-group differences may reflect real volumetric changes.

Recent functional imaging studies have shown that the insula plays a role in multimodal sensory integration, which is not surprising given its extensive connections to sensory processing regions of the cortex (Augustine, 1996). Simple audiovisual binding tasks have been shown to activate the insula bilaterally (Bischoff et al., 2007) and the left claustrum region (Olson et al., 2002; the claustrum shares the white matter fiber tracts of the extreme capsule with the insula). The right insula/claustrum re- gion was recently found to be activated in an audiovisual binding task that required conceptually relating a sound with a visual stimulus (Naghavi et al., 2007). This may be particularly relevant to the kind of cross-modal binding necessary in sign language. Naghavi et al. (p. 79) write: "Our findings indicate that the role of the claustrum/insula in [multisensory integration] ... involves integrative processes that require analysis of the content of stimuli." The insula is also implicated in tactile-vestibular integration (Bottini et al., 1995). It is important to note that spatial resolution of functional imaging methods generally cannot distinguish between insula or claustrum activation, which is unfortunate given that cytoarchitectonic studies suggest that despite sharing some connections, the two structures are developmentally and functionally distinct (Mufson and Mesulam, 1982).

Studies of visual-tactile integration have shown activation in the insula, albeit on different sides. Left insula activation was found in a study in which flat three-dimensional shapes were either felt by hand or viewed on a computer monitor (Banati et al., 2000), whereas right insula activation was observed in a task that involved feeling or viewing three-dimensional spheroids (Hadjikhani and Roland 1998). Sign language production can be regarded as a concept rich, visuotactile task, in which proprioceptive, tactile (contact with the body), and visual information must be integrated. Based on functional studies, such integration could be expected to engage the right insula, among other areas. In addition, the production of spatial language by both deaf and hearing signers engages right parietal cortices to a greater extent than for English speakers, which is hypothesized to be caused by the analog use of signing space to express spatial information (Emmorey et al., 2002, 2005). Thus, it is possible that signers may have different right hemisphere networks compared with speak- 
Table 4. Asymmetry indexes (AI) for insula and anterior and posterior lobules

\begin{tabular}{|c|c|c|c|c|c|}
\hline \multirow[b]{2}{*}{ Region } & \multicolumn{3}{|l|}{ Al group mean (SD) } & \multirow[b]{2}{*}{ Between-groups $p$ value $\left(F_{(2,63)}\right)$} & \multirow[b]{2}{*}{ Significant differences (Bonferroni $p$ value) } \\
\hline & Hearing $(H)$ & Deaf & Hearing signers (HS) & & \\
\hline Insula GM & $-0.001(0.035)$ & $0.052(0.042)^{*}$ & $0.033(0.042)^{*}$ & $<0.001(11.412)$ & $\begin{array}{l}\text { Deaf }>\mathrm{H}(<0.001) \\
\mathrm{HS}>\mathrm{H}(0.026)\end{array}$ \\
\hline Anterior lobule GM & $0.028(0.085)$ & $0.044(0.062)^{*}$ & $0.065(0.062)^{*}$ & ns & na \\
\hline Posterior lobule GM & $-0.058(0.134)^{\dagger}$ & $0.067(0.108)^{*}$ & $-0.030(0.092)$ & $0.001(7.901)$ & $\begin{array}{l}\text { Deaf }>H(0.001) \\
\text { Deaf }>\text { HS }(0.032)\end{array}$ \\
\hline Insula WM & $-0.679(0.204)^{*}$ & $-0.832(0.157)^{*}$ & $-0.730(0.310)^{*}$ & $0.052(3.107)$ & Deaf < $\mathrm{H}(0.050)$ \\
\hline Anterior lobule WM & $-0.542(0.210)^{*}$ & $-0.738(0.180)^{*}$ & $-0.634(0.321)^{*}$ & $0.015(4.494)$ & Deaf $<\mathrm{H}(0.012)$ \\
\hline Posterior lobule WM & $-0.962(0.306)^{*}$ & $-0.987(0.240)^{*}$ & $-0.915(0.377)^{*}$ & ns & na \\
\hline
\end{tabular}

Al formula: $(L-R) /[(L+R) / 2]$. GM, Gray matter; WM, white matter. ${ }^{*} p<0.01,{ }^{\dagger} p<0.05$, pairwise $t$ test.

A GRAY MATTER ASYMMETRY INDEX

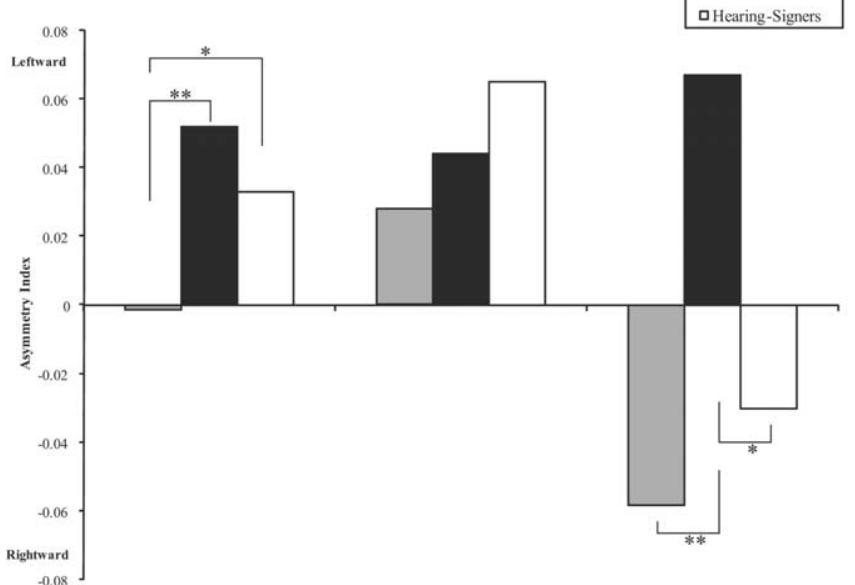

Insula GM Anterior Lobule GM

B WHITE MATTER ASYMMETRY INDEX

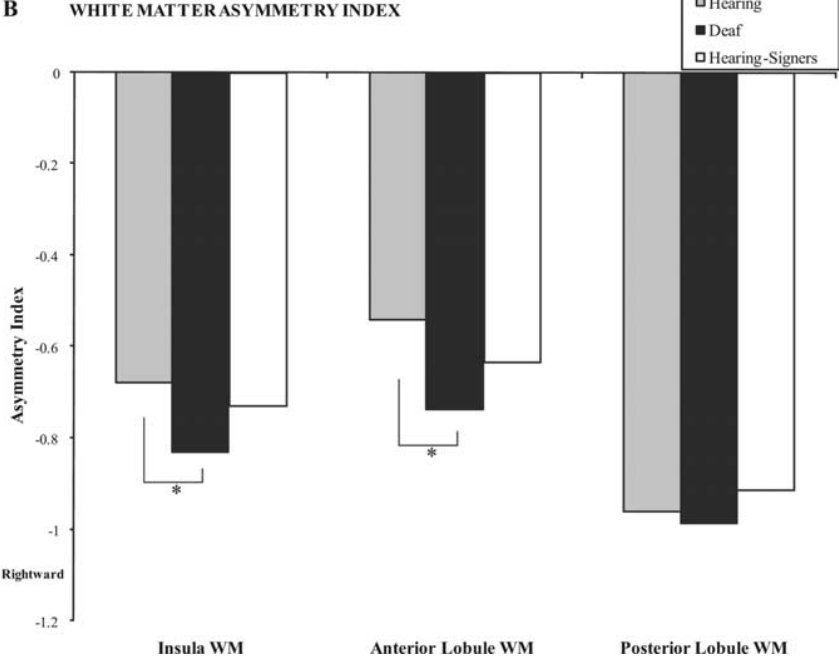

Figure 3. $\boldsymbol{A}$, Insula gray matter asymmetry index by region ( $t$ test, ${ }^{*} p<0.05$; ${ }^{* *} p<0.01$ ). $\boldsymbol{B}$, Insula white matter asymmetry index by region ( $t$ test, ${ }^{*} p<0.05$; ${ }^{* *} p<0.01$ ).

ers because of the unique use of space in sign language. Our finding of increased WM volume in the right insula of signers is consistent with a model of developmental enrichment, possibly leading to localized enhanced neuronal connectivity, resulting from the acquisition of ASL from an early age. Further functional imaging studies using appropriate, sign language-related tasks could confirm whether the right insula is an exceptionally important area for monitoring or integrating visual-motor aspects of sign language production.

We found that the GM of the left posterior insular lobule was increased in size in the deaf subjects compared with the two hearing groups. It is not clear why auditory deprivation would lead to increased gray matter in the insula. However, it is possible that deaf individuals' increased reliance on speech reading and/or their distinct speech might lead to an increase in cortical growth within the left posterior insula. MacSweeney et al. (2001) report activation in the left posterior insula for lip-reading for deaf but not for hearing individuals. The left insula has also been implicated in phonological processes in speech production (e.g., Blank et al., 2002; Shafto et al., 2007). Deaf individuals may recruit the left insula to a greater extent than hearing speakers because they rely more on visual-motoric representations of spoken phonology than on acoustic representations (e.g., Leybaert, 1993). Given that the posterior insula is primarily involved in somatosensory, visual, and motor functions (Makris et al., 2006), we speculate that differential reliance on visual and motor aspects of spoken language for deaf individuals is what leads to differences in gray matter volume in the left posterior insula.

Our study has some limitations. First, the large number of functions involving the insula provides many potential confounds for interpreting structurofunctional relationships. For example, the left insula is activated in a phonological working memory task in fully bilingual individuals but not in individuals whose second language expertise is measurably less than their first language (Chee et al., 2004). This means that bilingualism itself may affect the insula, and the only native bilingual group in our study is the group of hearing signers. Similarly, other group differences such as reading experience or visual attention might contribute to the observed anatomical differences within the insula. Second, the groups in our study are relatively small, particularly the hearing signers. Third, the delineation of the white matter of the insula including all of the extreme capsule, which by necessity we have to do, may include fiber tracks that are not directly related to the insula.

We have demonstrated that there are statistically significant volumetric differences in the GM and WM of the insula associated with hearing status and sign language experience. Given the wide range of cognitive functions in which the insula has a role, the interpretation of these differences is not straightforward. Nonetheless, based on the results from several functional imaging studies, we hypothesize that increased WM volumes observed in the right insula of deaf and hearing signers may be caused by enhanced connectivity in this region resulting from the increased reliance on cross-modal sensory integration in sign language compared with spoken language. We also suggest that increased GM volume in the posterior insula of deaf subjects could be related to reliance on visual speech reading and/or with more articulatory-based phonological representations of speech. These hypotheses can be tested with more detailed structural anatomy 
studies of connectivity within the insula region, and with functional studies using appropriate cross-modal integration tasks.

\section{References}

Ackermann H, Riecker A (2004) The contribution of the insula to motor aspects of speech production: A review and a hypothesis. Brain Lang 89:320-328.

Allen JS, Damasio H, Grabowski TJ, Bruss J, Zhang W (2003) Sexual dimorphism and asymmetries in the gray-white composition of the human cerebrum. Neuroimage 18:880-894.

Allen JS, Bruss J, Brown CK, Damasio H (2005a) Normal neuroanatomical variation due to age: the major lobes and a parcellation of the temporal lobe. Neurobiol Aging 26:1245-1260; discussion 1279-1282.

Allen JS, Bruss J, Brown CK, Damasio H (2005b) Methods for studying the aging brain: volumetric analyses versus VBM. Neurobiol Aging 26:1275-1278.

Augustine JR (1996) Circuitry and functional aspects of the insular lobe in primates including humans. Brain Res Brain Res Rev 22:229-244.

Bamiou DE, Musiek FE, Luxon LM (2003) The insula (Island of Reil) and its role in auditory processing: literature review. Brain Res Rev 42:143-154.

Banati RB, Goerres GW, Tjoa C, Aggleton JP, Grasby P (2000) The functional anatomy of visual-tactile integration in man: a study using positron emission tomography. Neuropsychologia 38:115-124.

Bavelier D, Dye MWG, Hauser PC (2006) Do deaf individuals see better? Trends Cogn Sci 10:512-518.

Bischoff M, Walter B, Blecker CR, Morgen K, Vaitl D, Sammer G (2007) Utilizing the ventriloquism-effect to investigate audio-visual binding. Neuropsychologia 45:578-586.

Blank SC, Scott SK, Murphy K, Warburton E, Wise RJS (2002) Speech production: Wernicke, Broca, and beyond. Brain 125:1829-1838.

Bottini G, Paulesu E, Sterzi R, Warburton E, Wise RJS, Vallar G, Frackowiak RSJ, Frith CD (1995) Modulation of conscious experience by peripheral sensory stimuli. Nature 376:778-781.

Cao Y, Whalen S, Huang J, Berger KL, DeLano MC (2003) Asymmetry of subsinsular anisotropy by in vivo diffusion tensor imaging. Hum Brain Mapp 20:82-90.

Chee MWL, Soon CS, Lee HL, Pallier C (2004) Left insula activation: A marker for language attainment in bilinguals. Proc Natl Acad Sci U S A 101:15265-15270.

Driver J, Noesselt T (2008) Multisensory interplay reveals crossmodal influences on "sensory-specific" brain regions, neural responses, and judgments. Neuron 57:11-23.

Dronkers NF (1996) A new brain region for coordinating speech articulation. Nature 384:159-161.

Emmorey K, Damasio H, McCullough S, Grabowski T, Ponto LLB, Hichwa RD, Bellugi U (2002) Neural systems underlying spatial language in American Sign Language. Neuroimage 17:812-824.

Emmorey K, Allen JS, Bruss J, Schenker N, Damasio H (2003) A morphometric analysis of auditory brain regions in congenitally deaf adults. Proc Natl Acad Sci U S A 100:10049-10054.

Emmorey K, Grabowski T, McCullough S, Ponto LLB, Hichwa RD, Damasio $H$ (2005) The neural correlates of spatial language in English and American Sign Language: a PET study with hearing bilinguals. Neuroimage 24:832-840.

Emmorey K, Korpics F, Petronio K (2008a) The use of visual feedback during signing: evidence from signers with impaired vision. J Deaf Stud Deaf Educ. Advance online publication. Retrieved October 15, 2008. doi:10.1093/deafed/enn020.

Emmorey K, Gertsberg N, Korpics F, Wright CE (2008b) The influence of visual feedback and register changes on sign language production: a kinematic study with deaf signers. Appl Psycholinguist, in press.
Flynn FG, Benson DF, Ardila A (1999) Anatomy of the insula-functional and clinical correlates. Aphasiology 13:55-78.

Frank RJ, Damasio H, Grabowski TJ (1997) Brainvox: An interactive, multimodal, visualization, and analysis system for neuroanatomical imaging. Neuroimage 5:13-30.

Grabowski TJ, Frank RJ, Szumski NR, Brown CK, Damasio H (2000) Validation of partial tissue segmentation of single-channel magnetic resonance images of the brain. Neuroimage 12:640-656.

Hadjikhani N, Roland PE (1998) Cross-modal transfer of information between the tactile and the visual representations in the human brain: a positron emission tomographic study. J Neurosci 18:1072-1084.

Holmes CJ, Hoge R, Collins L, Woods R, Toga AW, Evans AC (1998) Enhancement of MR images using registration for signal averaging. J Comput Assist Tomogr 22:324-333.

Kassubek J, Hickok G, Erhard P (2004) Involvement of classical anterior and posterior language areas in sign language production, as investigated by $4 \mathrm{~T}$ functional magnetic resonance imaging. Neurosci Lett 364:168-172.

Leybaert J (1993) Reading in the deaf: the roles of phonological codes. In: Psychological perspectives on deafness (Marschark M, Clark MD, eds). Hillsdale, NJ: Erlbaum.

MacSweeney M, Campbell R, Calvert GA, McGuire PK, David AS, Suckling J, Andrew C, Woll B, Brammer MJ (2001) Dispersed activation in the left temporal cortex for speech-reading in congenitally deaf people. Proc Biol Sci 268:451-457.

Majewska AK, Sur M (2006) Plasticity and specificity of cortical processing networks. Trends Neurosci 29:323-329.

Makris N, Goldstein JM, Kennedy D, Hodge SM, Caviness VS, Faraone SV Tsuang MT, Seidman LJ (2006) Decreased volume of left and total anterior insular lobule in schizophrenia. Schizophr Res 83:155-171.

Mesulam MM, Mufson EJ (1982a) Insula of the old world monkey. I: Architectonics in the insulo-orbito-temporal component of the paralimbic brain. J Comp Neurol 212:1-22.

Mesulam MM, Mufson EJ (1982b) Insula of the old world monkey. III: Efferent cortical output and comments on function. J Comp Neurol 212:38-52.

Mufson EJ, Mesulam MM (1982) Insula of the old world monkey. II: Afferent cortical input and comments on the claustrum. J Comp Neurol 212:23-37.

Naghavi HR, Eriksson J, Larsson A, Nyberg L (2007) The claustrum/insula region integrates conceptually related sounds and pictures. Neurosci Lett 422:77-80

Noesselt T, Shah NJ, Jäncke L (2003) Top-down and bottom-up modulation of language related areas-an fMRI study. BMC Neurosci 4:13.

Olson IR, Gatenby JC, Gore JC (2002) A comparison of bound and unbound audio-visual information processing in the human cerebral cortex. Brain Res Cogn Brain Res 14:129-138.

Saze T, Hirao K, Namiki C, Fukuyama H, Hayashi T, Murai T (2007) Insular volume reduction in schizophrenia. Eur Arch Psychiatry Clin Neurosci 257:473-479.

Shafto MA, Burke DM, Stamatakis EA, Tam PP, Tyler LK (2007) On the tip-of-the-tongue: neural correlates of increased word-finding failures in normal aging. J Cogn Neurosci 19:2060-2070.

Shelley BP, Trimble MR (2004) The insular lobe of Reil-its anatamicofunctional, behavioural and neuropsychiatric attributes in humans-a review. World J Biol Psychiatry 5:176-200.

Türe U, Yaçsargil DCH, Al-Mefty O, Yaçsargil MG (1999) Topographic anatomy of the insular region. J Neurosurg 90:720-733.

Woods RP, Cherry SR, Mazziotta JC (1992) A rapid automated algorithm for accurately aligning and reslicing PET images. J Comput Assist Tomogr 16:620-633. 